\title{
On the $\alpha$-particle semi-microscopic optical potential at low energies
}

\author{
Marilena Avrigeanu*, Faustin Laurentiu Roman, and Vlad Avrigeanu \\ "Horia Hulubei" National Institute for Physics and Nuclear Engineering \\ P.O.Box MG-6, Bucharest, Romania \\ E-mail: mavrig@ifin.nipne.ro
}

\section{Wolfram von Oertzen}

Freie Universität Berlin, Fachbereich Physics, and Hahn-Meitner-Institut

Glienicker Strasse 100, 14109 Berlin, Germany

E-mail: oertzen@hmi.de

\begin{abstract}
A semi-microscopic analysis based on the Double Folding Model (DFM) for the $\alpha$-particle elastic scattering on A 60 nuclei at energies below $20 \mathrm{MeV}$ has been carried out. The energydependent phenomenological imaginary part for this semi-microscopic optical model potential (OMP) was obtained, making use also of the dispersive correction to the microscopic real potential. This imaginary potential has then been introduced within a complete phenomenological analysis of the same data basis. A regional parameter set of the phenomenological OMP for low-energy $\alpha$-particles has thus been obtained for nuclei in the mass range $\mathrm{A} \sim 60$. Then it has been proved able to describe both scattering and $\alpha$-particle induced reaction data, through the statistical model analysis of most accurate data of $(\alpha, \mathrm{n})$ and total $\alpha$-reaction cross sections for ${ }^{48} \mathrm{Ti}$ and ${ }^{51} \mathrm{~V}$.
\end{abstract}

International Symposium on Nuclear Astrophysics - Nuclei in the Cosmos - IX

CERN, Geneva, Switzerland

25-30 June, 2006

\footnotetext{
${ }^{*}$ Speaker
} 


\section{Introduction}

The optical model potential (OMP) analyses for $\alpha$-particles at low energies pointed out two main questions which are still open, e.g. [1] and Refs. therein, namely (i) the OMP parameter sets obtained from $\alpha$-particle elastic scattering at high energies $(>80 \mathrm{MeV})[2]$ do not describe either the low-energy ( $<40 \mathrm{MeV}$ ) elastic scattering or the complete fusion data, and (ii) the statistical $\alpha$-particle emission is underestimated by the OMPs that account for elastic scattering on the (cold) ground-state nuclei. In the latter case, the need for new physics in potentials to describe nuclear de-excitation within the statistical model calculations was pointed out [3]. It was thus suggested that particle evaporation occurs from a transient nuclear stratosphere of the emitter nucleus, with a density that differs from cold nuclei [4] and which has not yet relaxed to the density profile expected for complete equilibration [5]. Therefore, effects due to changes of the nuclear density at a finite temperature have been considered within the double folding (DF) formalism [6] of the alpha-nucleus real part of the optical potential. Thus, it is shown that a temperature-dependent nuclear density distribution can be an important aspect to be included in statistical-model calculations even for temperatures $<2 \mathrm{MeV}$. At the same time, the high precision of recent measurements [7] of $\alpha$-particle elastic-scattering data makes possible the pointing out of additional features of the $\alpha$-particles scattering at low energy and, consequently, of further limits and possible improvement of global OMP parameters obtained previously through semi-microscopic analysis $[1,8]$ of the low-energy $\alpha$-particle elastic scattering. The involvement of these potentials for further description of both the scattering and emission of $\alpha$-particles is moreover discussed in the present work with reference to the mass region- A 60.

\section{2. $(\alpha, \alpha)$ semi-microscopic and phenomenological optical potential analyses at $A \sim 60$}

The previous semi-microscopic and phenomenological analyses of the $\alpha$-particle elastic scattering on A 100 nuclei at energies below $32 \mathrm{MeV}$ [6], based on the DF model (DFM), has been extended to nuclei within the astrophysically relevant iron peak, A 60. In the present work we focus on the analysis of experimental angular distributions on target nuclei ${ }^{50} \mathrm{Cr}$ and ${ }^{58,62} \mathrm{Ni}$ for $\alpha$-particle energies below $20 \mathrm{MeV}$ [9], more recent and also of first astrophysical interest, while next the energies up to $40-50 \mathrm{MeV}$ will be considered too. Within our two-step OMP approach [1], we determined first the parameters of an energy-dependent phenomenological imaginary part with a "parameter free" DF-real potential, by taking into account also the dispersive corrections to the microscopic real potential. Then a full phenomenological analysis of the same data has been carried out, with the general form of the optical potential with WoodsSaxon form factor also for the real part, and the imaginary part unchanged from the former semi-microscopic analysis. The advantage of having well settled already at least half of the usual OMP parameters increases obviously the accuracy of the local fit of data (Fig. 1). The corresponding average mass-, charge-, and energy-dependent OMP parameters, similar to those introduced by Nolte et al. [2] above $80 \mathrm{MeV}$, have been obtained in the end of this latter step. 

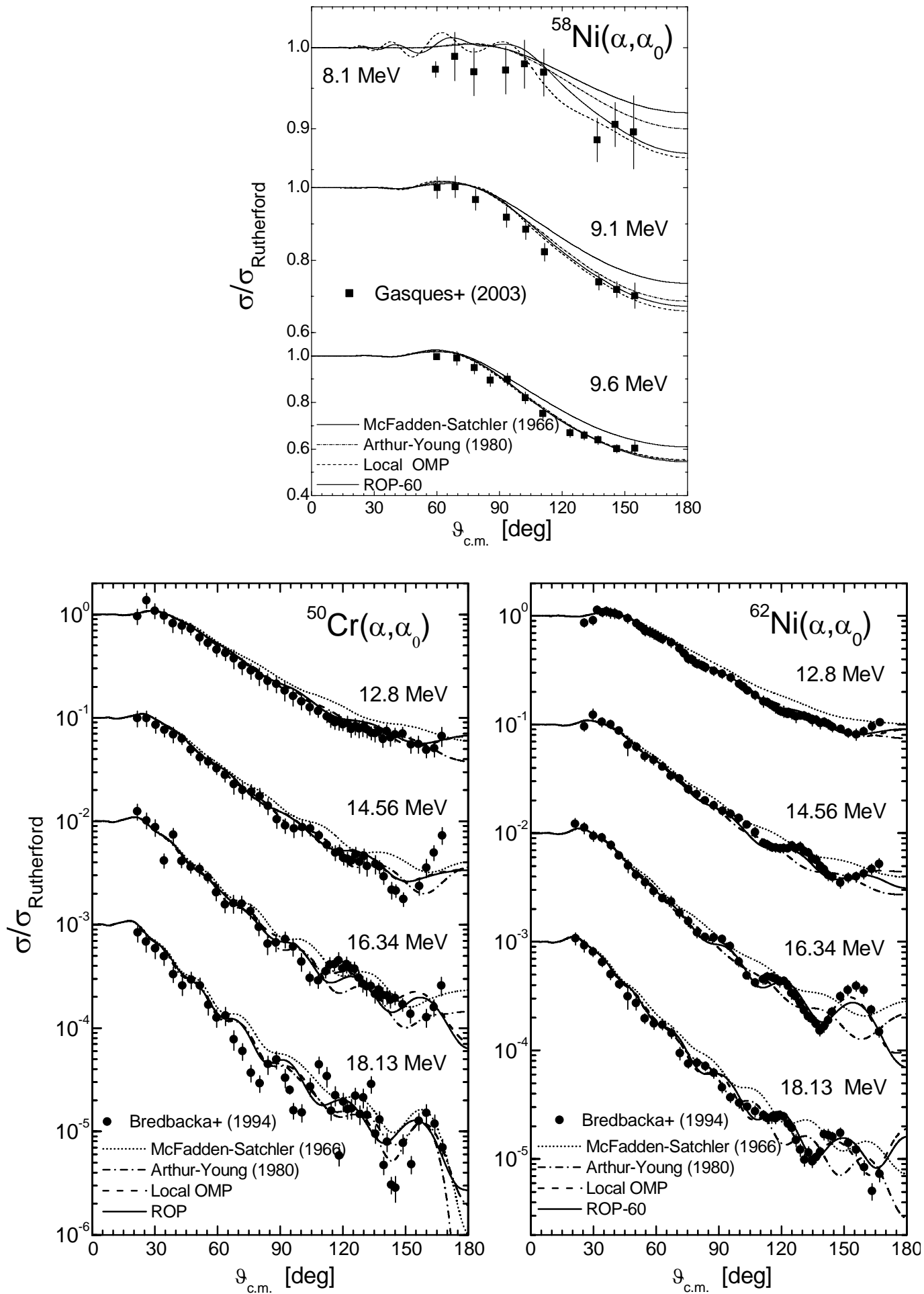

Figure 1: Comparison of measured [9] and calculated $\left(\alpha, \alpha_{0}\right)$ angular distributions on ${ }^{50} \mathrm{Cr}$ and ${ }^{58,62} \mathrm{Ni}$ using present local OMP (dashed curves), ROP (solid), and OMPs of Refs. [10,11] (dotted, dash-dotted).

Actually we looked for and found similar geometry parameters to the low-energy regional optical potential (ROP) for A 100 [1], except the volume imaginary potential reduced radius $r_{V}=1.25 \mathrm{fm}$, while the well depth expressions are as follows: 


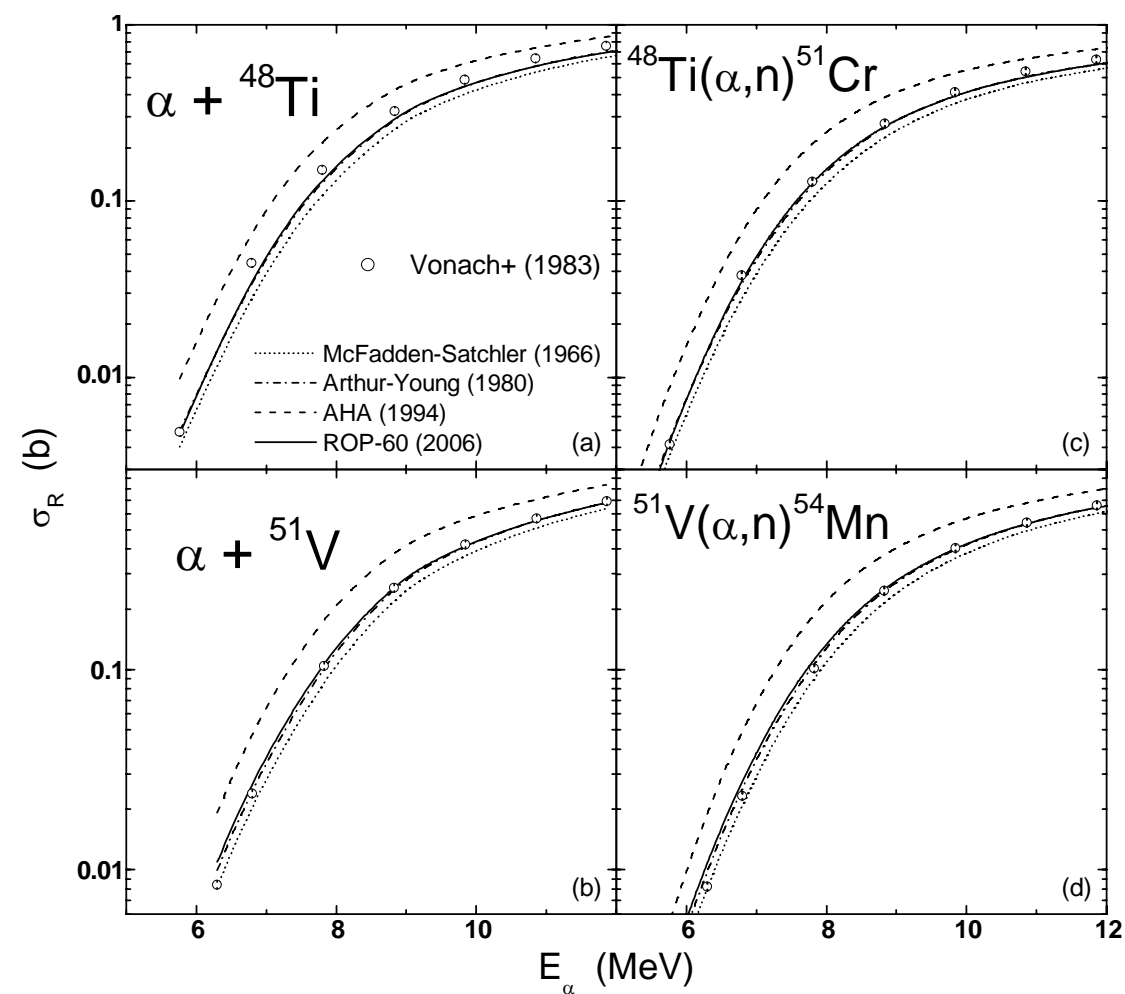

Figure 2: Comparison of measured [13] and calculated $(\alpha, n)$ and $\sigma_{\mathrm{R}}$ cross sections for ${ }^{48} \mathrm{Ti}$ and ${ }^{51} \mathrm{~V}$ using present ROP (solid) and OMPs of Refs. [10,11,12] (dotted, dash-dotted and dashed curves, respectively).

$$
\begin{aligned}
& V_{R}=\left\{\begin{array}{cc}
192.8+240.8 \eta, & E \leq 5.65 \mathrm{MeV} \\
204+240.8 \eta-2 E, & E>5.65 \mathrm{MeV}
\end{array}\right. \\
& W_{V}=\left\{\begin{array}{cc}
-8+49 \eta+(2.67-16.4 \eta) E, & E \leq 9 \mathrm{MeV} \\
7.9-98 \eta+0.9 E, & E>9 \mathrm{MeV}
\end{array}\right. \\
& W_{D}=\left\{\begin{array}{cc}
-4.65-26.5 \eta+(1.55+8.85 \eta) E, & E \leq 9 \mathrm{MeV} \\
13.6+53 \eta-0.476 E, & E>9 \mathrm{MeV}
\end{array}\right.
\end{aligned}
$$

where $\eta=(\mathrm{N}-\mathrm{Z}) / \mathrm{A}$ is the asymmetry parameter. The experimental data description provided by this ROP for A 60 and $\alpha$-particle energies below 20 is also shown in Fig. 1, in comparison with the predictions of the well-known global potentials of McFadden and Satchler [10] and Arthur and Young established within the analysis of fast neutron-induced reactions on ${ }^{54,56} \mathrm{Fe}$ [11]. Therefore we find that a rather suitable description of the analyzed data is provided by OMPs with real-potential diffuseness $a_{R}[1,10,11]$ notably lower than $a_{R}$-values which are needed in order to describe $\alpha$-particle emission from excited compound nuclei (e.g., Refs. [6,12]).

\section{3. $(\alpha, n)$ and total $\alpha$-reaction cross sections for ${ }^{48} \mathrm{Ti}$ and ${ }^{51} \mathrm{~V}$}

A key assessment of the ROP obtained for A 60 by the semi-microscopic and phenomenological analyses in the previous section has consisted in the analysis of the particularly accurate $(\alpha, n)$ and total $\alpha$-reaction cross sections for ${ }^{48} \mathrm{Ti}$ and ${ }^{51} \mathrm{~V}$ measured and respectively established by Vonach et al. [13]. The main points of these data are their 
description within about 10\% [13] by the parameter sets of McFadden and Satchler [10] and Arthur and Young [11] and an obvious overestimation by the OMP for $\alpha$-particle emission [12].

The Hauser-Feshbach statistical model calculations of these $(\alpha, n)$ reaction cross sections have been carried out similarly to the previous analysis of $(n, \alpha)$ reaction cross section for A 90 [6], except different optical potentials used in the incident and emergent $\alpha$-particle channels. A consistent local parameter set for the mass range A 60 was used, adopted recently [14] through an analysis of various independent data. The reaction cross sections (Fig. 2) calculated with the same OMP parameters finally involved in the discussion of the $\left(\alpha, \alpha_{0}\right)$ angular distributions (Fig. 1 ) show that both scattering and $\alpha$-induced reaction data are well described by the present ROP. Further work will be devoted to semi-microscopic description of $\alpha$-particle emission for A 60.

Acknowledgements. Most useful discussions with Prof. Helmut Leeb are gratefully acknowledged. Work supported in part by EURATOM-MEdC Fusion Association and MEdC Contract No. CEEX-05-D10-48.

\section{References}

[1] M. Avrigeanu, W. von Oertzen, A.J.M. Plompen and V. Avrigeanu, Optical model potentials for $\alpha$ particles scattering around the Coulomb barrier on A 100 nuclei, Nucl. Phys. A 723 (2003) 104.

[2] M. Nolte, H. Machner and J. Bojowald, Phys. Rev. C 36 (1987) 1312.

[3] G. La Rana et al., Phys. Rev. C 35 (1987) 373; G.D.J. Moses et al., Phys. Rev. C 36 (1987) 422.

[4] A.N. Antonov, J. Kanev, I.Zh. Petkov and M.V. Stoitsov, Il Nuovo Cimento A 101 (1989) 525.

[5] R. Lacey et al., Phys. Lett. B 191 (1987) 253; G. Bozzolo et al., Phys. Lett. B 219 (1989) 161.

[6] M. Avrigeanu, W. von Oertzen and V. Avrigeanu, On temperature dependence of the optical potential for alpha-particles at low energies, Nucl. Phys. A 764 (2006) 246.

[7] Zs. Fülöp, Gy. Gyürky, Z. Màtè, E. Samorjai, L. Zolnai, D. Galaviz et al., ${ }^{92} M o(\alpha, \alpha)^{92} M o$ scattering, the 92Mo-a optical potential, and the ${ }^{96} \mathrm{Ru}(\gamma, \alpha)^{92}$ Mo reaction rate at astrophysically relevant energie,s Phys. Rev. C 64 (2001) 065805; D. Galaviz, Zs. Fülöp, Gy. Gyürky, Z. Màtè, P. Mohr, T. Rauscher et al., Elastic $\alpha$-scattering on ${ }^{112} \mathrm{Sn}$ and ${ }^{124} \mathrm{Sn}$ at astrophysically relevant energies, Phys. Rev. C 71 (2005) 065802.

[8] M. Avrigeanu and V. Avrigeanu, Addendum to "Elastic $\alpha$-scattering on ${ }^{112} \mathrm{Sn}$ and ${ }^{124} \mathrm{Sn}$ at astrophysically relevant energies", Phys. Rev. C 73 (2006) 038801.

[9] L.R. Gasques et al., Phys. Rev. C 67 (2003) 024602; A. Bredbacka et al., Nucl. Phys. A 574 (1994) 397.

[10] L. McFadden and G.R. Satchler, Nucl. Phys. A 84 (1966) 177.

[11] E. Arthur and P.G. Young, LANL Report LA-8626-MS (ENDF-304), 1980.

[12] V. Avrigeanu, P.E. Hodgson and M. Avrigeanu, Global optical potentials for emitted alpha particles, Phys. Rev. C 49 (1994) 2136.

[13] H. Vonach, R.C. Haight and G. Winkler, Phys. Rev. C 28 (1994) 2278.

[14] V. Semkova, V. Avrigeanu, T. Glodariu, A.J. Koning, A.J.M. Plompen, D.L. Smith and S. Sudar, Nucl. Phys. A 730 (2004) 255. 